\title{
MANEJO ODONTOPEDIÁTRICO DE PACIENTE CON SINDROME DOWN: REPORTE DE CASO.
}

\section{PEDIATRIC DENTISTRY MANAGMENT OF PATIENTS WITH DOWN SINDROME: CASE REPORT}

\author{
Merchan Rueda Gabriela ${ }^{1} *$ Romero Velarde Mariela $^{2}$ \\ ${ }^{1}$ Residente de la Especialidad de Odontopediatría de la Univesridad San Martín de Porres.Perú. \\ ${ }^{2}$ Docente de la Especialidad de Odontopediatría de la Univesridad San Martín de Porres.Perú. \\ *gabriela.mechanrueda@gmail.com
}

\begin{abstract}
Resumen
El Síndrome Down es una anomalía congénita que se caracteriza por presentar hipotonía muscular generalizada, cambios neurológicos con deterioro intelectual y una diversidad de problemas sistémicos, además, de tendencia a presentar problemas dentales. Los niños con discapacidades, incluidas las condiciones que afectan el comportamiento y la cognición, a menudo tienen limitaciones para la atención odontológica requiriendo el uso de técnicas de manejo de conducta farmacológicas; por lo que es muy importante reforzar la salud oral desde edades tempranas. Se reporta el caso de un niño de cinco años cuatro meses de edad de sexo masculino que acudió a la Clínica Odontológica Especializada de la Universidad de San Martin de Porres para atención odontopediátrica. Se planteó tratamiento multidisciplinario; se destaca el uso de técnicas de manejo de conducta no farmacológicas para el paciente.
\end{abstract}

Palabras clave: Síndrome Down, Manejo de conducta, Odontopediatría, Caries Dental .

\begin{abstract}
Down Syndrome is a congenital anomaly that is characterized by the generalized hypotonic, neurological changes with intellectual impairment and risk to systemic issues, in addition, tendency to dental issues. Children with disabilities, including conditions that affect behavior and cognition, often present limitations for dental care requiring the use of pharmacological behavior management techniques). It is important to consider the oral health component from early ages. The present case report corresponds to a 5-year-old male patient that attended to the Specialized Pediatric Dentistry Clinic of the San Martin de Porres University. A multidisciplinary treatment was proposed during the treatment planned; highlighting the usage of non-pharmacological behavior management techniques for the patient.
\end{abstract}

Key words: Down Syndrome, Behavior Management, Pediatric Dentistry, Dental Caries. .

\section{INTRODUCCIÓN}

El Síndrome de Down (SD), también conocido como trisomía del par 21, es una anomalía autosómica congénita caracterizada por hipotonía muscular generalizada, cambios neurológicos con deterioro intelectual y una diversidad de problemas sistémicos; tales como problemas de audición y de vista, hipotiroidismo, apnea obstructiva del sueño, enfermedad celíaca, convulsiones e inestabilidad atlantoaxial; estos problemas de salud no tratados pueden dar lugar a un aumento de los problemas de conducta, alteraciones adicionales en la cognición / aprendizaje y la disminución de la calidad de vida. ${ }^{1,2}$ Por otro lado, existe cierta aversión de estos pacientes al entorno tradicional del consultorio odontológico lo que resulta en problemas conductuales y además genera una barrera adicional a la atención. ${ }^{2}$ Un enfoque conductual puede trascender la situación disminuyendo la resistencia física por parte del paciente, permitiendo de esta manera realizar los tratamientos y que aprenda a tolerar situaciones incómodas. Como parte de las técnicas de manejo de conducta no farmacológicas encontramos algunas alternativas como: desensibilización, modelamiento, control de voz, refuerzo positivo. ${ }^{3}$ La caries de aparición temprana (CAT) se define como la presencia de una o más lesiones de caries cavitadas o no cavitadas en niños menores de 6 años de edad. ${ }^{4}$ Por ello algunos estudios han informado que los pacientes con SD tienen menos prevalencia de caries que aquellos que no la presentan. La literatura lo atribuye a factores tales como: el patrón de erupción (erupción tardía de los dientes por lo que 
están expuestos a factores etiológicos de caries por menos tiempo); alta prevalencia de bruxismo (superficies oclusales planas que facilitan la auto-limpieza, hipersalivación y la higiene oral). ${ }^{1,2}$ Sin embargo, no existe evidencia científica que apoye la hipótesis de que las personas con SD presenten una prevalencia menor de caries ya que la mayoría de trabajos científicos no presentan un control adecuado de variables, lo cual debilitaría esta afirmación., ${ }^{5,6}$

Es importante considerar que el bruxismo también es bastante común en esta población, desde edades muy tempranas. Los pacientes con SD tienen ansiedad crónica, el sistema nervioso poco desarrollado, maloclusión y disfunción de la ATM, debido a la hipotonía, hiperflexibilidad y laxitud de los ligamentos de soporte. ${ }^{1}$ El objetivo de este reporte de caso clínico es brindar información sobre las técnicas de manejo de conducta no farmacológicas usadas durante el tratamiento odontopediátrico en un paciente con Síndrome Down que presentó caries de aparición temprana.

\section{REPORTE DE CASO}

Paciente de cinco años y cuatro meses de edad de sexo masculino que acude a consulta a la clínica especializada de la USMP con el diagnóstico de Síndrome Down y su madre refiere que tiene "dientes picados". En los antecedentes personales patológicos la madre relató que su menor hijo tiene problemas auditivos en el oído izquierdo, presenta pie plano por lo que usa zapatos ortopédicos. Características extraorales: presenta braquicefalia, tercio medio facial disminuido, nariz en silla de montar. Su lengua da la impresión de ser anormalmente grande a causa de la hipotonía muscular y de la posición anterior y baja en la boca. Figura 1. Al examen estomatológico se encuentra en los tejidos duros, con fosas y fisuras profundas en las piezas dentales 55, 65, 75, 85; lesiones no cavitadas activas en las piezas 55(v), 54(v), 53(v), 63(v), 64(v), 65(v), 75(vl), 74(v), 84(v) 85(v); lesiones cavitadas activas en las piezas 54(o), 64(od), 74(od). Pieza 84 pulpitis reversible.; bruxismo y conducta II según Frankl levemente negativo. Figuras 2-3.

Se utilizó técnica de sensibilización para incrementar los tiempos de trabajo, la complejidad de los mismos y la aceptación del paciente. En las primeras citas se usó la técnica de restricción física o conocida actualmente como estabilización protectora activa; es decir, técnica rodilla a rodilla con la ayuda de la madre para la atención del niño y se inició con sesiones de fluorterapia con Fluoruro de Sodio al $5 \%$ en presentación Barniz. Como adecuación de medio se realizó la restauración de la pieza 74 mediante Tratamiento Restaurador Atraumático (TRA) con ionómero de alta viscosidad al cabo de cinco meses aproximadamente se observó clínicamente la pérdida parcial del material como se puede apreciar en la radiografía (Figura 5), razón por la cual se tomó la decisión de restaurar con ionómero de vidrio modificado con resina preservando el material anterior que se encontraba próximo a la cámara pulpar. En el caso de

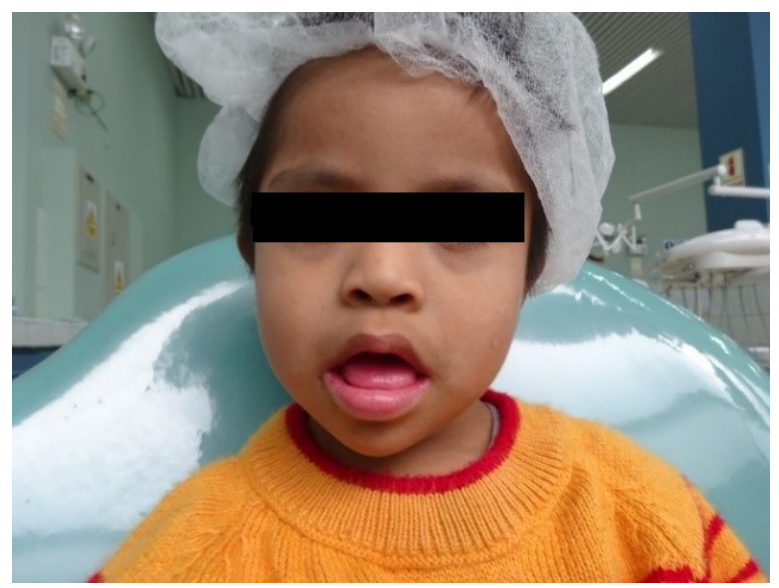

Fig. 1. Fotografía extraoral frontal

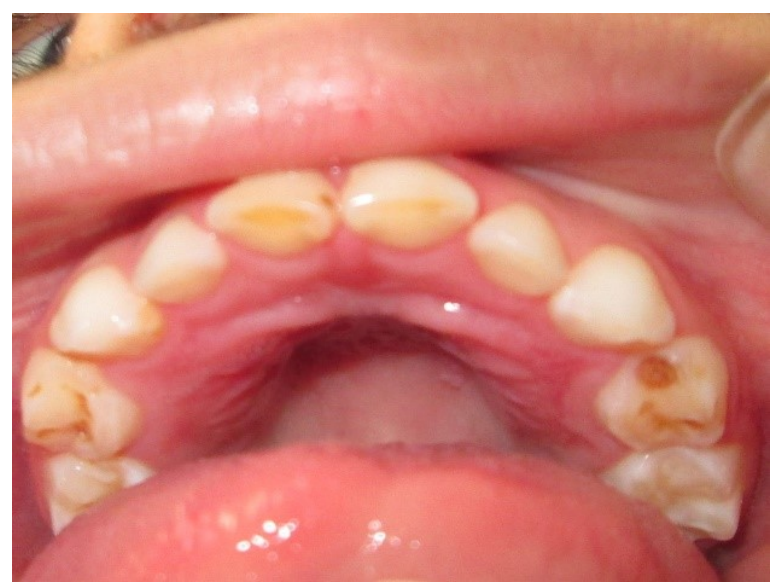

Fig. 2. Fotografía oclusal superior

los tratamientos para sellantes de fosas y fisuras profundas se optó por el material de ionómero de vidrio modificado con resina para las siguientes piezas dentales: 55, 65, 75, 85. El mismo material se utilizó también para las lesiones cavitadas activas de las piezas 54(o), 64(od). Durante cada procedimiento se explicó a la madre del menor sobre ciertas conductas negativas de su menor hijo; y que se usaría restricción física o estabilización protectora pasiva (Papoose Board) por la seguridad personal y del paciente ante los diferentes procedimientos que requerían el uso de anestesia local, como fue el caso de la pieza 84 que requirió el tratamiento pulpar de pulpotomía. Mediante el respectivo control radiográfico se observó que esta pieza presentó imagen radiopaca compatible con material de obturación de pulpotomía sin la presencia de alteraciones; por esta razón, se rehabilitó la pieza 84 con corona de acero, la elección de este tipo de corona se hizo para evitar el desgaste por presentar bruxismo y posterior a su colocación se realizaron sus respectivos controles. (Figura 8-9) 


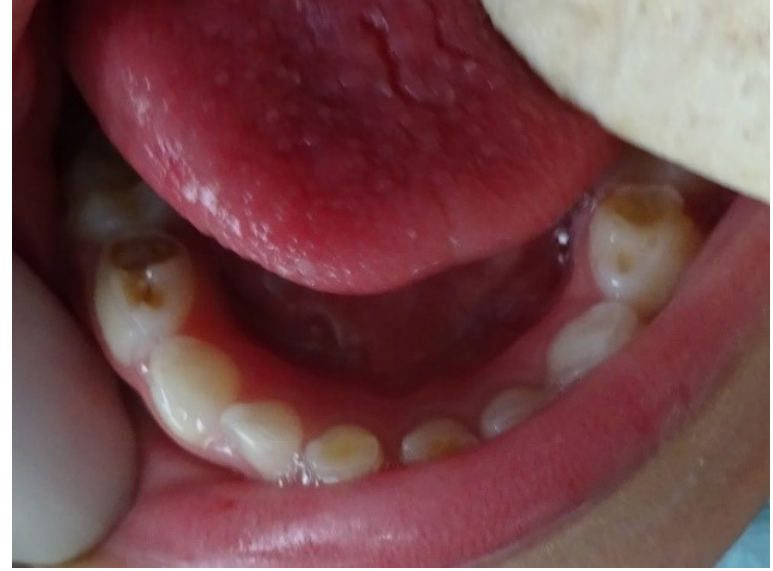

Fig. 3. Fotografía oclusal inferior

Cabe recalcar que después de finalizado cada tratamiento se procuró mantener como rutina, dedicar un tiempo para interactuar con el paciente y su madre, utilizando estrategias lúdicas lo que ayudó a entablar un ambiente de confianza y a su vez realizar un refuerzo positivo al tratamiento. Al finalizar los tratamientos planteados se observó que hubo mayor aceptación y colaboración por parte del niño y de sus padres por lo que el pronóstico de este paciente se consideró favorable por terminar modificando su conducta de manera positiva.

\section{DISCUSIÓN}

Existen estudios en los que se menciona que la mayoría de los padres de los niños con discapacidad consideraban que sus hijos tenían una necesidad para el cuidado dental curativo en lugar de los procedimientos de prevención. ${ }^{7,8}$

Para los padres de pacientes con discapacidades; las razones más frecuentes para no buscar atención dental fueron: el costo, el miedo/ ansiedad, la disponibilidad y capacidad del personal odontológico. Reportaban visitas al dentista con más frecuencia para tratamientos de emergencia, extracciones, restauraciones que los niños normales. ${ }^{9}$ En este caso los padres del paciente, manifestaron las mismas razones por las que no asistieron antes a consulta y además indicaron el temor a la conducta y resistencia negativa de su hijo a la consulta odontológica. Existen dos grupos de técnicas de manejo de conducta usados en niños con conducta inadecuada en el consultorio dental: Dentro del primer grupo están las técnicas de modificación de la conducta, en las que se pretende la desensibilización. En el segundo grupo entrarían las técnicas restrictivas o de estabilización protectora, destinadas a la inmovilización del paciente, las cuales son muy útiles en pacientes con un retardo intelectual profundo. ${ }^{10}$ Dichas técnicas se aplicaron en este caso debido a la falta de cooperación y movimientos involuntarios por parte del paciente, que durante las primeras citas se mostró poco

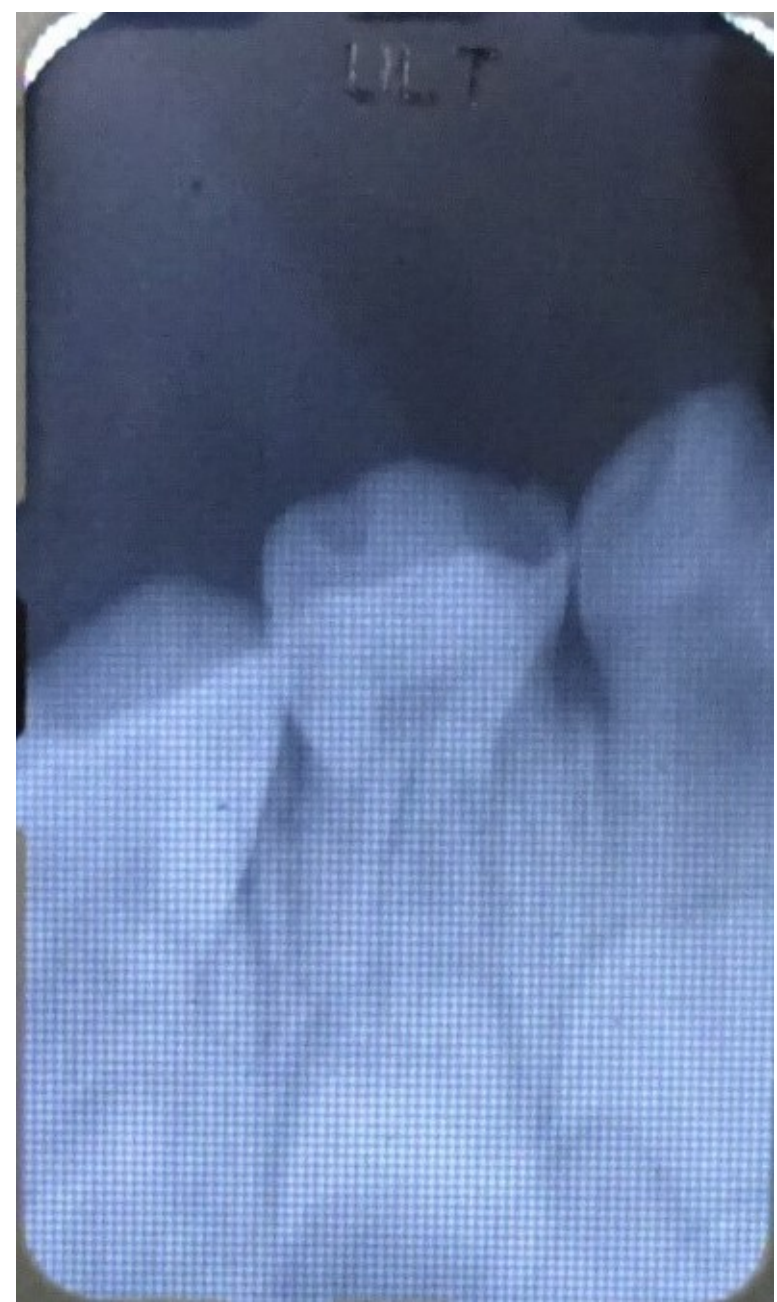

Fig. 4. . Radiografía pieza 74.

colaborador, y que al término de los tratamientos modificó su conducta siendo mucho más cooperador. En relación al problema de caries dental Quijano et al. en el año 2005 realizaron un estudio en dos Centros Educativos ubicados en el Cono Norte de Lima Metropolitana. La muestra fue de 92 niños, 42 con Síndrome Down y 50 niños normales en edades comprendidas entre los 3 a 6 años. Se utilizó el criterio de diagnóstico de la OMS (ceo-d) obteniendo como resultado que los niños con síndrome Down presentaron el $70 \%$ a diferencia de los niños normales con un $42 \%$ de prevalencia de caries. Sin embargo, en este estudio no se evaluaron las lesiones no cavitadas en ninguna de las dos poblaciones y además se sugiere que la población de estudio de niños sin SD tendría acceso al servicio odontológico. ${ }^{11}$ Contrario a esto la evidencia científica sugiere que los individuos con SD tienen menos prevalencia de caries que las personas sin SD; esta evidencia puede ser debilitada por la ausencia de control de los factores de riesgo.

Duda et al. en el año 2015 encontró que las personas 


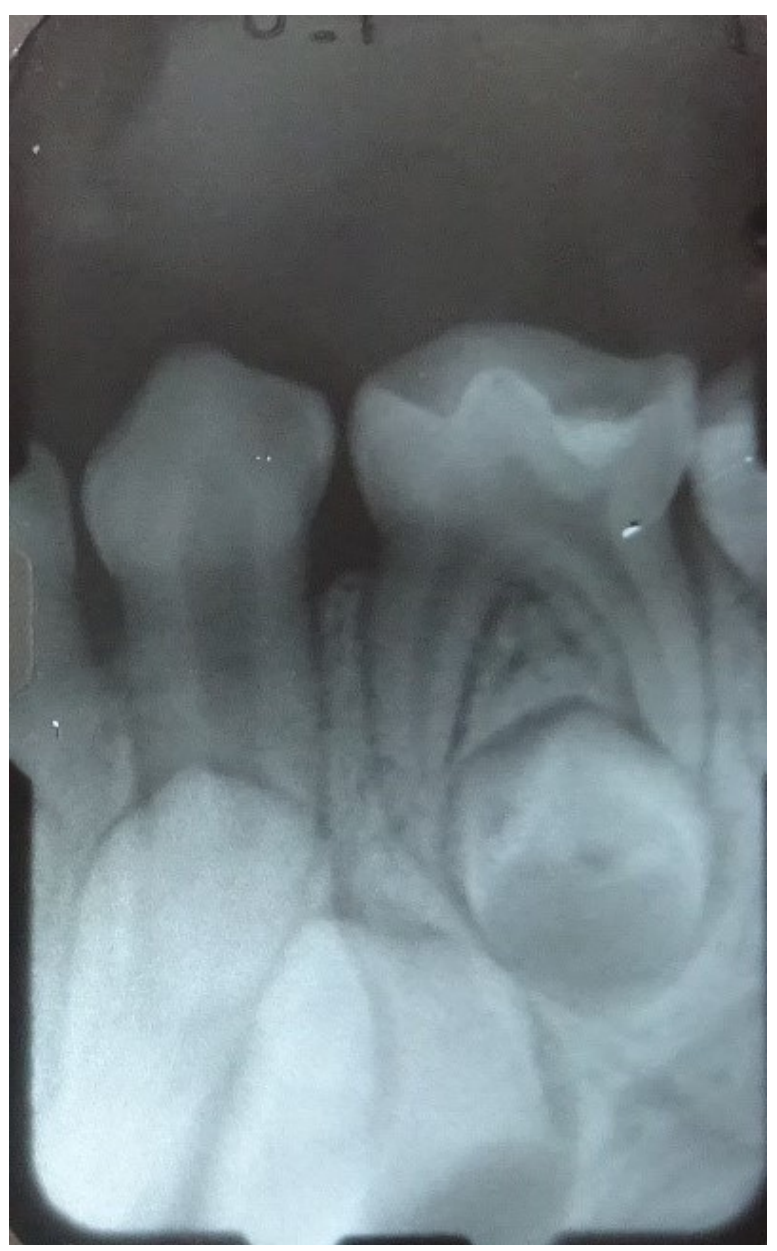

Fig. 5. Radiografía de la pieza 74 .

con síndrome Down, especialmente los niños que toman medicamentos con frecuencia para los síntomas de la sinusitis, otitis, amigdalitis y otras infecciones respiratorias comunes entre esta población; generalmente son prescritos con medicamentos pediátricos que contienen un alto nivel de azúcar en su composición, lo que resulta en un riesgo cariogénico en estos individuos. Esta es una variable que puede influir en la prevalencia o la experiencia de caries entre los individuos con SD. Se necesitan más estudios observacionales con tamaños de muestra más grandes, una correcta adecuación entre los casos y controles, y un mejor control de los factores de riesgo como la medicación, los hábitos dietéticos y la exposición a aplicaciones de flúor para confirmar esta evidencia. ${ }^{5}$ De igual manera Moreira et al. en el año 2015 concluye que no existe evidencia científica que apoye la hipótesis que las personas con Síndrome Down tienen una experiencia de caries menor a los individuos no sindrómicos. ${ }^{6}$ En este caso se observó que el paciente presentó lesiones de caries en diversos estadíos obteniéndose como diagnóstico: Caries de Aparición Temprana, lo cual corrobora lo expresado por la literatura

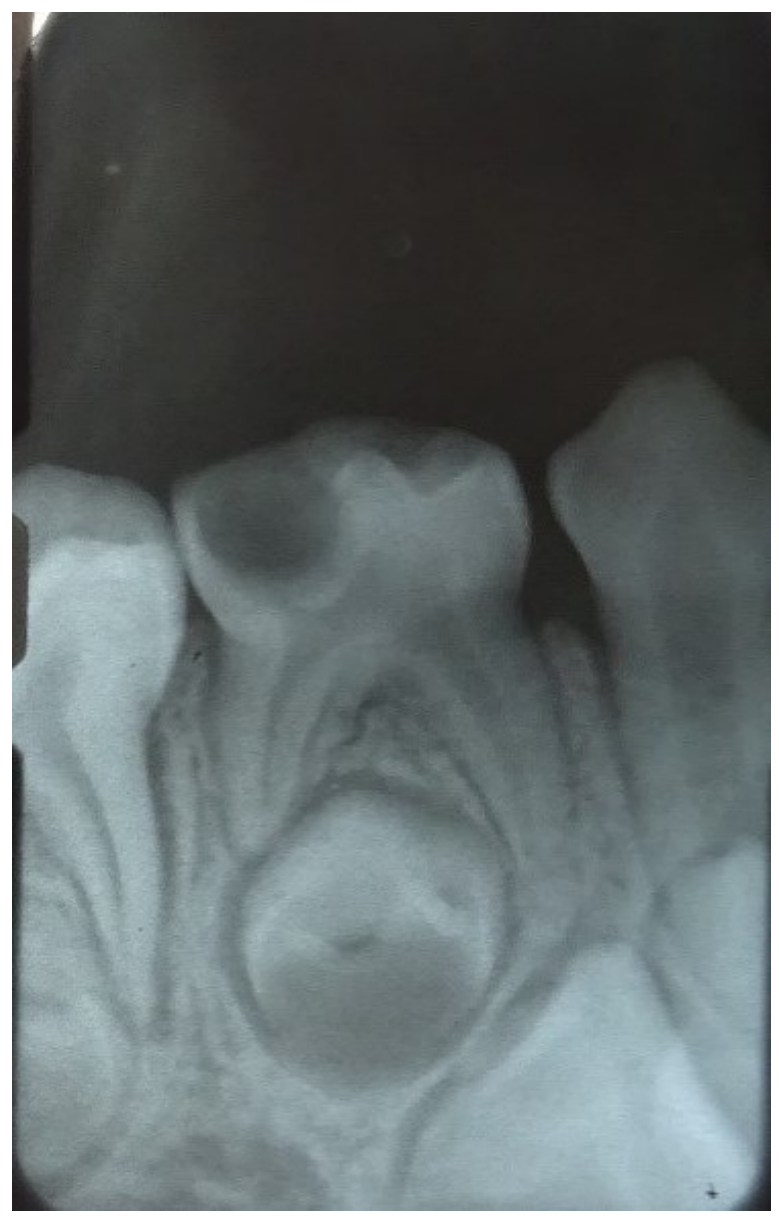

Fig. 6. Radiografía de la pieza 84.

científica. En la actualidad la Caries de Aparición Temprana es considerada una enfermedad de salud pública que afecta a una gran cantidad de niños. Su prevalencia varía de una población a otra. Entre los factores de riesgo a considerar están: dieta, higiene, acceso a servicios de salud, condiciones socioeconómicas y de educación de los padres o tutores. ${ }^{12}$

El papel de la higiene bucal parece ser decisivo en la prevención de la caries dental en los niños con SD. Mientras que los niños sin SD pueden realizar los procedimientos de higiene oral por sí solos o tener mejor destreza para realizarla, los niños con Síndrome Down dependen de sus padres o personas que están a su cuidado. Por lo tanto, la salud oral de los niños con Síndrome Down depende en gran medida de la cooperación y el esfuerzo de sus padres o tutores. Así que el enfoque preventivo debería ser dirigido a sus padres o tutores. $^{6}$

Por otra parte, Poulsen et al. llevaron a cabo un estudio en el que compararon la retención de sellantes a base de resina con sellantes de ionómero de vidrio, y se encontró que, después de 6 meses, el 90,09\% de los selladores a base de resina se mantuvieron por completo, $6,75 \%$ fueron retenidos 


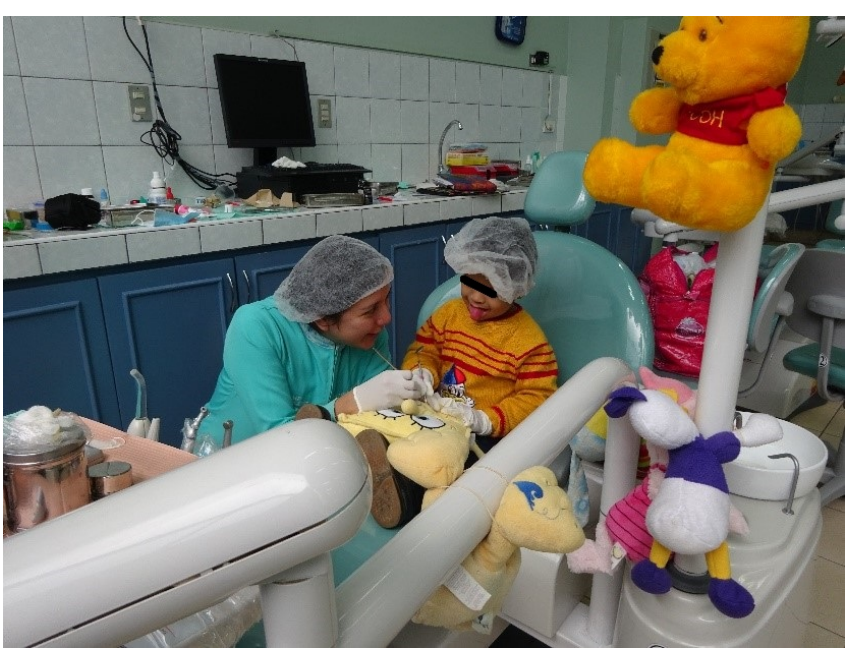

Fig. 7. Fotografía técnica de manejo de conducta de modelamiento.

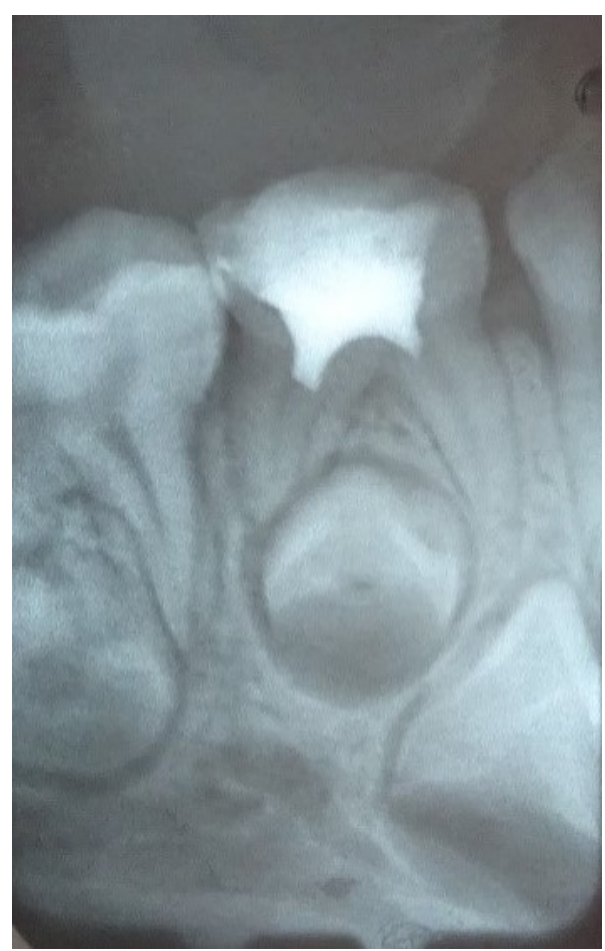

Fig. 8. Radiografía de la pieza 84 control del tratamiento de pulpotomía.

parcialmente, y $3,15 \%$ se había perdido por completo. $^{13}$ Forss et al, también obtuvieron resultados similares después de comparar la retención de un sellante a base de resina, y de un cemento de ionómero de vidrio. Los resultados fueron 10,3\% de los selladores de ionómero de vidrio, y $45,5 \%$ de los selladores con base de resina estaban totalmente presentes, mostrando una diferencia estadísticamente significativa de retención. ${ }^{14}$ Estos resultados se corroboran

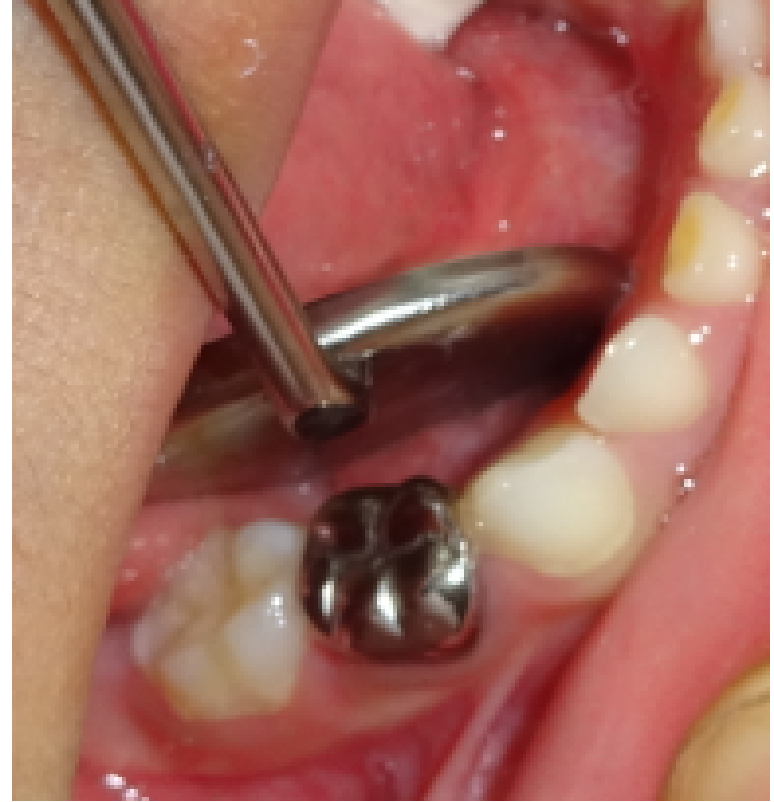

Fig. 9. . Fotografía clínica de la corona en la pieza 84.

con lo encontrado en este caso, que se aplicó ionómero modificado con resina observándose una buena retención tanto como sellante como material restaurador. Sin embargo, se debe considerar que falta longevidad a los estudios sobre materiales dentales en este tipo de pacientes. Además de tomar en consideración las diferentes alteraciones funcionales que presentan los pacientes con SD como es el caso de la parafunción de bruxismo. Como recomendación se detalla los siguientes procedimientos que han demostrado su eficacia para establecer armonía en las relaciones pacienteodontopediatra, reduciendo la ansiedad:

1. Dar un breve paseo por el consultorio antes de intentar el tratamiento. Presentar al paciente al personal del equipo asistencial y así se reducirá el temor del paciente a lo "desconocido".

2.Comunicarse con términos sencillos. Asegurarse que las explicaciones sean comprendidas preguntando a los pacientes si tienen alguna duda que resolver.

3. Brindar solamente una instrucción cada vez.

4.Escuchar atentamente al paciente.

5. Realizar sesiones cortas.

6. Programar la atención del paciente para horas tempranas del día, cuando el odontólogo, su equipo asistencial y el paciente están menos fatigados. 10

Los pacientes con SD plantean desafíos que requieren una capacitación especializada del personal odontopediátrico. Incluir dentro de los cursos de posgrado este tipo de experiencia es importante para que el profesional pueda otorgar la atención que demanda el paciente con SD. Una educación temprana, puede ayudar a mantener la salud, evitando que se 


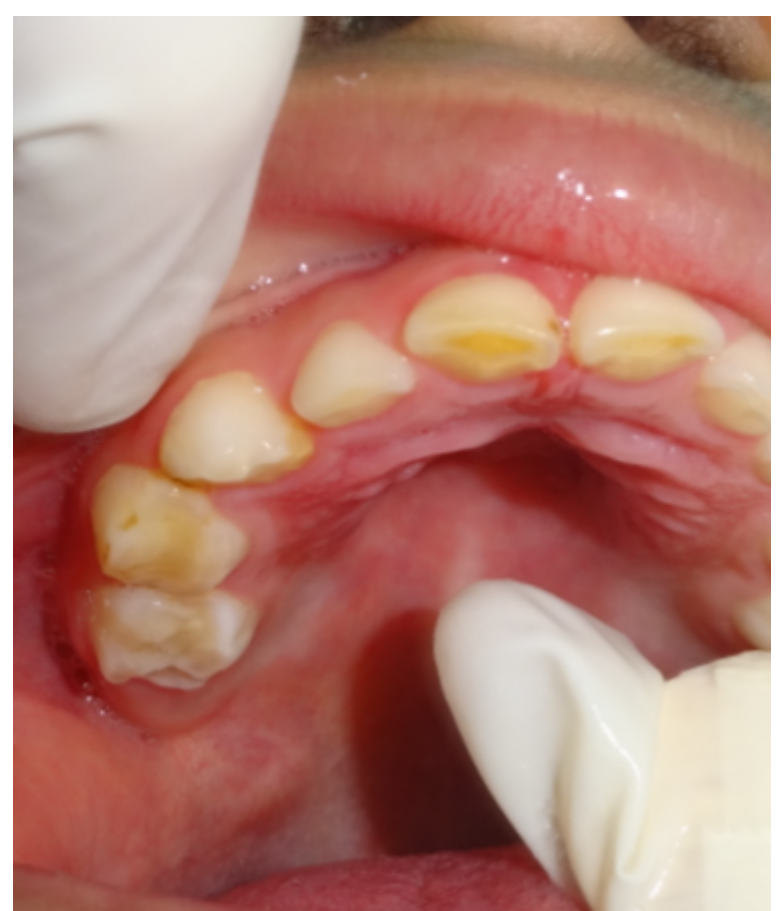

Fig. 10. Set fotográfico final. (Hemiarcadas) Hemiarcada superior lateral derecha.

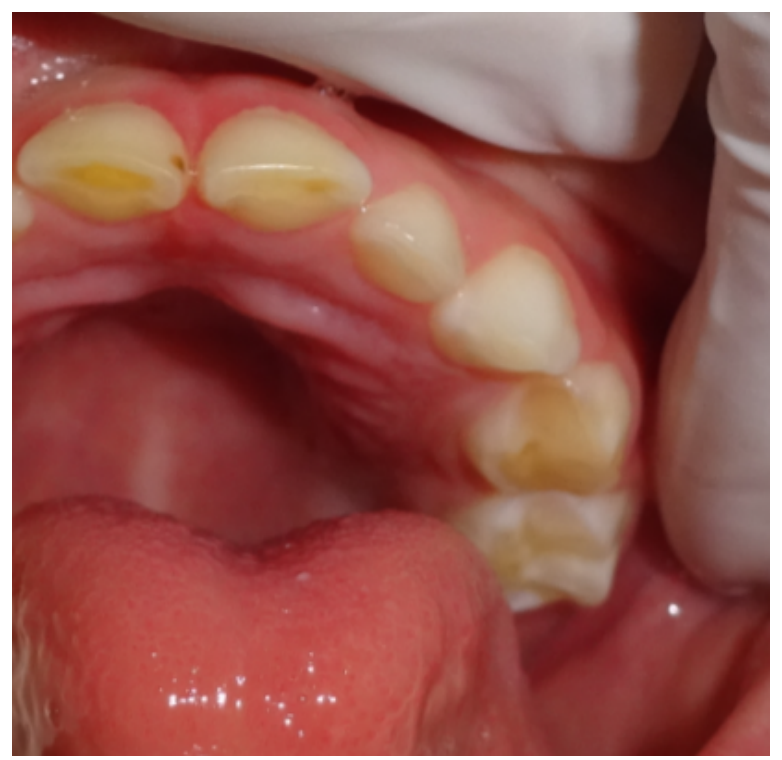

Fig. 11. Hemiarcada superior lateral izquierda

requiera llegar a tratamientos invasivos.

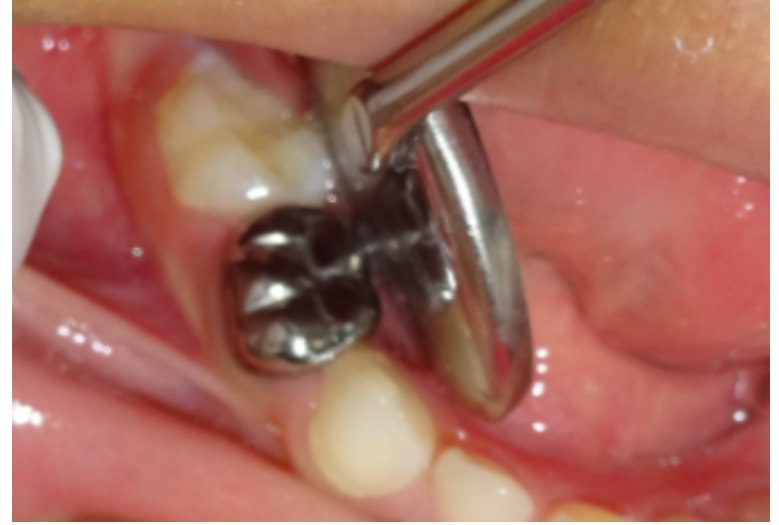

Fig. 12. Hemiarcada inferior lateral derecha

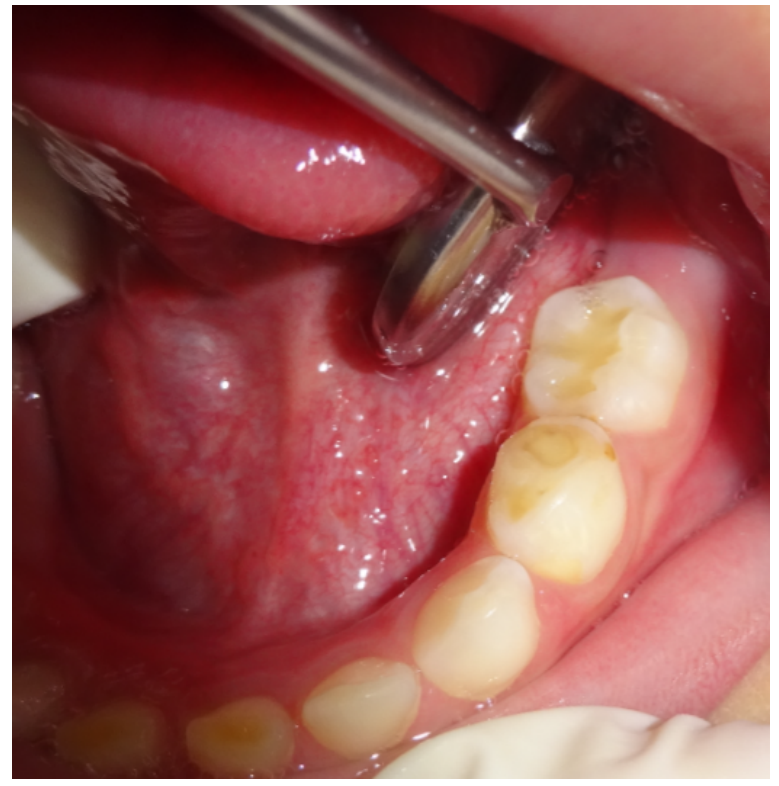

Fig. 13. Hemiarcada inferior lateral izquierda

\section{Referencias}

1 Macho V, Coelho A, Areias C, Macedo P, Andrade D. Craniofacial Features and Specific Oral Characteristics of Down Syndrome Children. Acta Port Pediatr. 2014; 13(2):408-11.

2 Norwood K, Slayton R. Oral Health Care for Children With Developmental Disabilities. Pediatr Dent 2013; 131:614-9.

3 Kemp F. Alternatives: a review of non-pharmacologic approaches to Increasing the cooperation of patients with special needs to Inherently unpleasant dental procedures. J Dent Child. 2005; (2):88-108.

4 American Academy of Pediatric Dentistry. Symposium on the prevention of oral disease in children and adolescents. Chicago, Ill; November 11-12, 2005: Conference papers. Pediatr Dent 2006; 28(2).96-8. 
5 Duda T, Lopes G, Castro C, Paiva S, Almeida J, Borges A. Association between Dental Caries and Down Syndrome: A Systematic Review and Meta-Analysis. Journal Pone 2015; 10(6): 1-11.

6 Moreira M, Shwertner C, Jardin J, Hashizume L. Dental caries in individuals with Down syndrome: a systematic review. Int J Clin Pediatr Dent 2016; 26: 3-12.

7 Newacheck P, Mcmanus M, Fox H, Hung Y, Halfon N. Access to health care for children with special health care needs. Pediatrics 2000; 105 : 760-6.

8 Gordon S, Dionne R, Synder J. Dental fear and anxiety as a barrier to accessing oral health care among patients with special health care needs. Spec Care Dent 1998; 18: 88-92.

9 Shiyama M, S Mutawa, Honkala E, Honkala S. Parental perceptions of dental visits and access to dental care among disabled schoolchildren in Kuwait. Odontostomatol Trop. 38(149):34-42.

10 Casillas E. Consideraciones en el manejo del paciente con Síndrome Down Odontología Actual.2014; (1):1-16.

11 Quijano G, Díaz M. Caries dental en niños pre-escolares con síndrome de Down. Rev Estomatol Herediana 2005; 15 (2): $128-132$.

12 Fernandes B, Pereira T, Souza D, Ramos-Jorge, Marques L, Ramos J. Severity of Dental Caries and Quality of Life for Toddlers and Their Families. Pediatr Dent. 2017;39(2):118123.

13 Navarro M, Coelho S, Molina G, Villena R. Tratamiento Restaraudor Atraumático: Actualidades y perspectivas. Rev Assoc Cir Dent 2015;69(3):289-301.

14 Flório F, Pereira A, Meneghim C, Ramacciato J. Evaluation of non-invasive treatment applied to occlusal surfaces. J Dent Child. 2001; 68:326-31.

Recibido: 30 de Enero de 2017.

Aceptado: 21 de Noviembre de 2017. 
